\title{
Altered expression of genes involved in brain energy metabolism as adaptive responses in rats exposed to chronic variable stress; changes in cortical level of glucogenic and neuroactive amino acids
}

\author{
MARIOLA HERBET ${ }^{1}$, DOROTA NATORSKA-CHOMICKA ${ }^{1}$, AGNIESZKA KORGA ${ }^{1}$, MARTA OSTROWSKA ${ }^{1}$, \\ MAGDALENA IZDEBSKA ${ }^{1}$, MONIKA GAWROŃSKA-GRZYWACZ ${ }^{1}$, IWONA PIĄTKOWSKA-CHMIEL ${ }^{1}$, \\ KAMIL PAWŁOWSKI ${ }^{1}$, BRYGIDA ŚLASKA ${ }^{2}$, EWA POLESZAK ${ }^{3}$ and JAROSŁAW DUDKA ${ }^{1}$ \\ ${ }^{1}$ Chair and Department of Toxicology, Medical University of Lublin, 20-090 Lublin; \\ ${ }^{2}$ Department of Biological Bases of Animal Production, University of Life Sciences in Lublin, 20-950 Lublin; \\ ${ }^{3}$ Department of Applied Pharmacy, Medical University of Lublin, 20-093 Lublin, Poland
}

Received July 29, 2018; Accepted December 18, 2018

DOI: $10.3892 / \mathrm{mmr} .2019 .9865$

\begin{abstract}
Brain metabolism is closely associated with neuronal activity and enables the accurate synthesis and function of neurotransmitters. Although previous studies have demonstrated that chronic stress is associated with the overproduction of reactive oxygen species (ROS), which leads to oxidative stress and the disruption of glucose metabolism, the molecular mechanisms and cerebral gluconeogenesis in depression have not yet been completely elucidated. In order to examine this subject, the present study evaluated changes in the expression of selected genes involved in the glycolytic pathway and the levels of glucogenic and neuroactive amino acids in the brain of rats exposed to chronic variable stress. Male Wistar rats (50-55 days old, weighing 200-250 g) were divided into two groups: control and stressed, and the rats in the stressed group were exposed to stress conditions for 40 days. Depressive-like states were observed and recorded by measuring the body weight and forced swim test (FST). The mRNA levels of Slc2a3 (coding GLUT3) and Tfam (activator of mitochondrial transcription and a participant in mitochondrial genome replication) were markedly increased, while a decrease in the expression of $L d h b$ and $G A P D H$ was also observed. These modifications were associated with the redirection of glucose metabolism to appropriate defensive pathways under chronic stress conditions, and an increased ability to maintain mitochondrial function as potential adaptive responses. A marked reduction of glucogenic and neuroactive amino acids levels indicate the support of energy metabolism
\end{abstract}

Correspondence to: Dr Mariola Herbet, Chair and Department of Toxicology, Medical University of Lublin, Jaczewskiego 8b, 20-090 Lublin, Poland

E-mail: mariola.herbet@umlub.pl

Key words: depression, oxidative stress, gene expression, glucose metabolism, amino acids by stimulation of the gluconeogenesis pathway. The findings of the present study provide a novel insight into the molecular and biochemical events that impact the development of depression under chronic stress conditions, and they may identify novel targets for therapeutic intervention.

\section{Introduction}

Chronic or recurring stress is associated with overproduction of reactive oxygen species (ROS) and reactive nitrogen species (RNS), highly unstable molecules with unpaired electrons, leading to oxidative stress and development of depression through various biological change (1-3). Oxidative stress causes progressive oxidative damage to lipids, proteins and DNA in neurons and impairs synaptic function. The neurons are especially vulnerable to ROS because neuronal activity increases oxygen utilization for energy production. The neuronal activity is closely related to brain metabolism and enables the proper synthesis of neurotransmitters, primarily glutamate and $\gamma$-aminobutyric acid (GABA) (4-6). Because entry of neuroactive compounds into the brain is highly restricted by the blood-brain barrier (BBB), these compounds must be synthesized from glucose (5). The brain has high energy requirements; excitatory neurotransmission accounts for most of the energy requirements at the cortical level. The high demand for energy is mainly achieved by the production of ATP during metabolism of glucose or oxidative phosphorylation in the mitochondria (6-8). The impaired of glucose metabolism results in decreased pyruvate production, which in turn can lead to mitochondrial DNA (mtDNA) mutations, and disorders in the mitochondrial respiratory chain function, mitochondrial defense systems and hence impaired energy metabolism, and can lead to impaired neuroplasticity and apoptosis (1). Thus, the brain under chronic stress show deficits in glucose metabolism, so alternative energy sources may help to prevent the cell death. Studies showed that when the brain cannot catabolize glucose efficiently, it may become reliant upon amino acid oxidation for energy production $(9,10)$. 
In the long period of time, effective mechanisms take place, which entail the transcriptional activation of genes and gene networks that function to control glucose homeostasis. In this context, glucose may regulate metabolic genes by modulating the activity of nuclear factors toward their target genes (11). Thus the molecular basis for physiological and pathological regulation of glucose metabolism, as potential adaptive responses in mitochondrial injury, can be determined at the level of transcription. Due to the restrictive permeability of the $\mathrm{BBB}$, the brain relies heavily upon glucose transporters for the delivery of key nutrients (12). Several glucose transporters have been identified in the brain, of which GLUT3, encoded by Slc $2 a 3$ is of major importance; it ensures that neurons receive a constant supply of glucose even when interstitial glucose concentrations are low (13). Glyceraldehyde 3-phosphate dehydrogenase (GAPDH) is an enzyme that plays an important role in energy metabolism; in the glycolysis process it catalyzes the reversible phosphorylation of glyceraldehyde-3-phosphate (G3P) to 1,3-bisphosphoglycerate (BPG) using $\mathrm{NAD}^{+}$as a cofactor (14). Lactate dehydrogenase (LDH) is the enzyme catalyzing the final step of the anaerobic metabolic pathway, glycolysis. LDH is a tetrameric enzyme made up of two different subunits $\mathrm{A}$ and $\mathrm{B}$, encoded by the LDHA and LDHB genes, respectively. LDHA catalyzes the conversion of pyruvate to lactate with concomitant inter-conversion of $\mathrm{NADH}$ to $\mathrm{NAD}^{+}$, while LDHB catalyzes the conversion of lactate to pyruvate. Mitochondrial transcription factor A (TFAM) is a major mtDNA-binding protein that packages it into nucleoprotein complexes, called nucleoids (15). TFAM has been shown to regulate transcription through specific binding to the promoter region and the copy number of mtDNA (16).

The in vivo studies have shown that the acute stress changes amino acid metabolism $(17,18)$. Under chronic stress conditions, the major aim of protein catabolism is to provide the glucogenic amino acids that serve as substrates for endogenous glucose production. The glucogenic amino acids, especially alanine and glutamine, can be converted into glucose in an enzymatic process of gluconeogenesis. The gluconeogenesis pathway is normally present in the liver, kidney, and intestine. However, the emerging reports show an evidence that gluconeogenic activity can also occur in brain, although the studies on cerebral gluconeogenesis are limited (19). In the hypermetabolic/stress state, gluconeogenesis increases dramatically and in proportion to the degree of the insult to increase the supply of glucose (the major fuel of reparation). Glucose is the only fuel that can be utilized by hypoxic tissues (anaerobic glycolysis) (20). This pathway found is becoming more recognized as an important alternative glucose source for neurons, specifically in the pathological conditions $(19,21)$. Amino acids also play an important role as the transmitters of synaptic excitation and the modulators of synaptic function in the central nervous system and they can serve a special role as the precursors for neurotransmitter synthesis (neuroactive amino acids) (22).

The disruption of pathways of glucose delivery and metabolism in cells exposed to chronic stress may lead to debilitation of brain disease through various biological changes; however, molecular mechanism and cerebral gluconeogenesis in depression are not established. In this study, we evaluated the changes in expression of selected genes involved in glycolytic pathway and the levels of glucogenic and neuroactive amino acids in the brain of rats exposed to chronic variable stress (CVS). In an attempt to mimic the excessive human day-to-day stress, several animal models have been developed. CVS, a well-validated animal model, has been used widely for studying clinical depression. The method, which multiple stressors relies on the ability of a sequence of relatively mild stressors, may lead to behavioral changes. According to in vivo studies, CVS model of depression, based on a 40 day treatment procedure with variables stressors involved, has high validity, since a large number of recent publications have confirmed that CVS induces behavioral and neurochemical changes in animals that are similar to the symptoms and presumed neurochemical changes accompanying depression in humans $(4,23,24)$. This protocol, unlike other protocols of chronic stress which use only one stressor, uses variable stressors, which prevents adaptation to stress. Studies have shown that 40-day variable stress causes alterations in feeding behavior and disturbs neurotransmission in the rodent brain (23). Moreover, it was proved that animals submitted to CVS may present some effects, such as increased adrenals after just 2 weeks of stress, while behavioral and neurochemical changes here may need more time to develop (23).

\section{Materials and methods}

Animals. The experiment was performed using male Wistar rats which were approximately 6 weeks old and weighed 200-250 g at the time of arrival. The rodents were purchased from a licensed breeder (Brwinów, Poland) and were housed in standard rectangular polypropylene cages with free access to standard diet and water. The standard laboratory conditions included maintaining a constant temperature $\left(20 \pm 2^{\circ} \mathrm{C}\right)$ and a $12 \mathrm{~h}$ day/12 $\mathrm{h}$ night cycle as well as constant environment (humidity, noise). The study design was approved by the Local Ethics Committee on Animal Experimentation of the Medical University of Lublin (no. 12/2015). The procedures were also performed in accordance with obligatory Polish and European standards related to the experimental studies on animals (Act from January 15, 2015 on the Protection of Animals Used for Scientific or Educational Purposes; Directive 2010/63/eu of the European Parliament and of the council of 22 September 2010 on the protection of animals used for scientific purposes).

CVS model of depression. A CVS protocol was based on the description of Gamaro et al having regard to a few modifications $(4,23)$. A variable-stressor paradigm was used in the study because the different stressors used diminish adaptation to stress (4). To avoid predictability, rats were exposed to these stressors not at the same time each day. A specification has been presented in detail in part of our published study (2). In brief, two groups of rats, each consisted of 10 randomly selected animals, were used in the study. Rodents in the control (CTL) group were kept undisturbed in their colony cages (5 rats in each cage of dimensions $65 \times 25 \mathrm{~cm}, 18 \mathrm{~cm}$-high), while those of the stressed group (CVS) were subjected to different external stressors for 40 days. The procedure implied using the following stress agents: food deprivation lasting 
Table I. Stress factors applied during the chronic variable stress procedure. Data adapted from Herbet et al (2).

\begin{tabular}{|c|c|c|}
\hline Day & Stress factor & Time of duration \\
\hline 1 & Water deprivation & $24 \mathrm{~h}$ \\
\hline 2 & Food deprivation & $24 \mathrm{~h}$ \\
\hline 3 & Isolation & $24 \mathrm{~h}$ \\
\hline 4 & Isolation & $24 \mathrm{~h}$ \\
\hline 5 & Isolation & $24 \mathrm{~h}$ \\
\hline 6 & Flashing light & $3 \mathrm{~h}$ \\
\hline 7 & Food deprivation & $24 \mathrm{~h}$ \\
\hline 8 & Forced swimming & $10 \mathrm{~min}$ \\
\hline 9 & Restraint & $1 \mathrm{~h}$ \\
\hline 10 & Water deprivation & $24 \mathrm{~h}$ \\
\hline 11 & No stressor & - \\
\hline 12 & No stressor & - \\
\hline 13 & Restraint and cold & $2 \mathrm{~h}$ \\
\hline 14 & Flashing light & $2.5 \mathrm{~h}$ \\
\hline 15 & Food deprivation & $24 \mathrm{~h}$ \\
\hline 16 & Forced swimming & $15 \mathrm{~min}$ \\
\hline 17 & Isolation & $24 \mathrm{~h}$ \\
\hline 18 & Isolation & $24 \mathrm{~h}$ \\
\hline 19 & Isolation & $24 \mathrm{~h}$ \\
\hline 20 & Water deprivation & $24 \mathrm{~h}$ \\
\hline 21 & Food deprivation & $24 \mathrm{~h}$ \\
\hline 22 & Flashing light & $3 \mathrm{~h}$ \\
\hline 23 & Restraint & $2 \mathrm{~h}$ \\
\hline 24 & Isolation & $24 \mathrm{~h}$ \\
\hline 25 & Isolation & $24 \mathrm{~h}$ \\
\hline 26 & Restraint and cold & $1.5 \mathrm{~h}$ \\
\hline 27 & Forced swimming & $10 \mathrm{~min}$ \\
\hline 28 & Flashing light & $3.5 \mathrm{~h}$ \\
\hline 29 & No stressor & - \\
\hline 30 & Food deprivation & $24 \mathrm{~h}$ \\
\hline 31 & Restraint & $3 \mathrm{~h}$ \\
\hline 32 & Flashing light & $2 \mathrm{~h}$ \\
\hline 33 & Water deprivation & $24 \mathrm{~h}$ \\
\hline 34 & Restraint and cold & $2 \mathrm{~h}$ \\
\hline 35 & Forced swimming & $15 \mathrm{~min}$ \\
\hline 36 & Isolation & $24 \mathrm{~h}$ \\
\hline 37 & Isolation & $24 \mathrm{~h}$ \\
\hline 38 & No stressor & - \\
\hline 39 & Flashing light & $3 \mathrm{~h}$ \\
\hline 40 & Forced swimming & $10 \mathrm{~min}$ \\
\hline
\end{tabular}

$24 \mathrm{~h}$, water deprivation lasting $24 \mathrm{~h}$, restraint lasting 1-3 h at room temperature or $1.5-2 \mathrm{~h}$ at $4^{\circ} \mathrm{C}$, forced swimming for 10 or $15 \mathrm{~min}$, flashing light for $120-210 \mathrm{~min}$, and isolation (2-3 days). The names of stressors including time of application are presented in Table I. In the end of the experiment, the rats were sacrificed by decapitation at $24 \mathrm{~h}$ following the last stressor action. The brain sample from each animal was obtained, washed with $20 \mathrm{ml}$ of saline and stored at $-75^{\circ} \mathrm{C}$ until use for further analyses.
Forced swimming test. Forced swim test (FST) was performed according to Porsolt et al (25), in order to confirm the ability of stressors an indicative of depressive behavior. Rats were individually placed in a glass cylinder $(40 \mathrm{~cm}$ tall, $25 \mathrm{~cm}$ in diameter) containing water at $22-23^{\circ} \mathrm{C}$ to a height of $30 \mathrm{~cm}$. Water in the tank was changed after each rat swimming test section. According to the procedure, for the first exposure, rats were placed in the water for $15 \mathrm{~min}$, so in our experiment, the last stressor of CVS counted as pre-test session. Twenty-four $h$ later, rodents were placed in the water again for a 5 min test session, and the immobility time was recorded.

Reverse transcription-quantitative polymerase chain reaction (RT-qPCR) analysis. The samples of isolated hippocampus were rinsed with $20 \mu \mathrm{l}$ of saline and stored at $-75^{\circ} \mathrm{C}$ before the process of isolation of RNA. According to the manufacturer's instructions, the nucleic acid was separated from $30 \mathrm{mg}$ of tissue using Syngen Tissue RNA Mini kit (Syngen Biotech, Poland) and reverse transcription was performed using NG dART RT-PCR kit (EURx, Poland). The assessment of the chosen genes expression (Slc2a3, GAPDH, Ldha, Ldhb and Tfam) was performed using a quantitative real-time PCR (qPCR) method. The relative expression of genes was measured with the $\Delta \Delta \mathrm{Ct}$ method, using Hprt (Mn00446968_m1) as an endogenous control. The reaction was carried out in octuplicate by q-PCR using the SmartChip Real-Time PCR System (WaferGen Biosystems, Inc., Fremont, CA, USA), and TaqMan Fast Universal PCR Master Mix (2X) (Applied BioSystems; Thermo Fisher Scientific, Inc., Waltham, MA, USA) according to manufacturer's instructions. Sample quality screening based on amplification, $\mathrm{Tm}$, and $\mathrm{Ct}$ values was performed to remove any outlier data points before $\Delta \Delta \mathrm{Ct}$ calculation and to determine fold change in mRNA levels. The data was presented as $R Q$ value $\left(R Q=2^{-\Delta \Delta C t}\right)$.

Amino acids analysis. Reagents used to prepare ninhydrin solution: Ninhydrin, hydrindantine dihydrate, ethylene glycol monomethyl ether, $4 \mathrm{M}$ acetate buffer $\mathrm{pH}$ 5.6. Reagents used for preparation of lithium-based buffers are: citric acid monohydrate, lithium citrate tetrahydrate, lithium chloride, thiodiglycol, sodium azide and lithium hydroxide monohydrate. Physiological amino acid standard, Asn+Gln standard and all reagents mentioned above (used for amino acid analysis) were obtained from Ingos (Prague, Czech Republic). Sulphosalicylic acid (SSA) were purchased from POCH S.A. (Gliwice, Poland).

To assess the concentrations of the glucogenic and neuroactive amino acids, approximately $100 \mathrm{mg}$ of prefrontal cortex of rat brain from each animal from each group were deproteinized and homogenized in 6\% SSA in lithium citrate buffer ( $\mathrm{pH} 2.8$ ) in 1:10 ratio. The homogenised samples were centrifuged $20 \mathrm{~min}$ at $12,000 \mathrm{rpm}$. The concentration of free amino acids in the obtained supernatants was determined using an automatic analyzer Ingos AAA 500. Amino acids were separated by the ion-exchange chromatography using analytic column Ostion LG FA (Ingos) with five lithium-based buffers ( $\mathrm{pH} 2.6,3.1,3.35,4.05$ and 4.65). The original software Clarity version 6.1.0.130 (DataApex Ltd., Prague, Czech Republic) was used to estimate the concentrations of free amino acids (26). 
Statistical analysis. Data was analyzed by STATISTICA v. 10 application (StaftSoft, Cracow, Poland). The results are presented as mean \pm SEM and expressed as percentage of CTL group. Comparisons between groups were done using an unpaired Student's t-test and $\mathrm{P}<0.05$ was considered to indicate a statistically significant difference.

\section{Results}

Body weight. Body weight was measured at 1, 10, 20, 30 days of the procedure and at the end of the experiment. Our study indicated that stressed rats have presented a lower body weight than unstressed [day 1: CTL, 285.7 $\pm 2.504 \mathrm{~g}$; CVS, 287.0 $\pm 5.175 \mathrm{~g} ; \mathrm{P}=0.823 ; t=0.226$ (18); $\mathrm{F}=4.272$; day 10: $\mathrm{CTL}$, $335.00 \pm 2.687$ g; CVS, 335.0 \pm 6.708 g; $\mathrm{P}>0.999$; $\mathrm{t}=0.00$ (18); $\mathrm{F}=6.231$; day 20: CTL, $418.00 \pm 4.89 \mathrm{~g}$; CVS, $344.00 \pm 4.76 \mathrm{~g}$; $\mathrm{P}<0.001 ; \mathrm{t}=10.832$ (18); $\mathrm{F}=1.059$; day 30: $\mathrm{CTL}, 465.00 \pm 7.03 \mathrm{~g}$; CVS, 357.00 $\pm 6.50 \mathrm{~g} ; \mathrm{P}<0.001 ; \mathrm{t}=11.273$ (18); $\mathrm{F}=1,168$; day 40: CTL, $524.5 \pm 10.71 \mathrm{~g}$; CVS, $411.0 \pm 7.219 \mathrm{~g} ; \mathrm{P}<0.01 ; t=8.788$ (18); $\mathrm{F}=2.201]$. The effect of CVS on body weight is shown in Fig. 1 .

Forced swimming test. After 40 days of stress procedure, forced swimming test was performed. As shown in Fig. 2, immobility time was increased in animals exposed to CVS conditions, when compared to the CTL group, indicating that these animals have presented depressive behavior [CTL, $63 \pm 1.88 \mathrm{sec}$; CVS, $78 \pm 2.376$ sec; $\mathrm{P}<0.01 ; t=4.951$ (18); $\mathrm{F}=1.597]$.

The level of mRNA expression. The expression levels of selected genes involved in brain glucose metabolism were examined (Table II). Sample variation was accounted for by comparison to expression levels of $\mathrm{Hprt}$, which is a housekeeping gene responsible for metabolism of nucleotide. Expression of mRNA was measured in reference to the CTL group, where the expression level is estimated as $R Q=1$. The mRNA levels of Slc2a3 [CTL, 1.0 \pm 0.057 ; CVS, 1.236 \pm 0.104 ; $\mathrm{P}<0.05 ; t=2.1$ (57); $\mathrm{F}=2.522]$ and Tfam [CTL, 1.0 $\pm 0.064 ; \mathrm{CVS}$, $1.303 \pm 0.94 ; \mathrm{P}<0.05 ; t=2.583$ (60); $\mathrm{F}=2.285$ ] were significantly increased, while an decrease in the expression of $L d h b$ [CTL, $1.0 \pm 0.043$; CVS, $0.685 \pm 0.028 ; \mathrm{P}<0.01 ; t=5.533$ (60); $\mathrm{F}=1.904]$ and $G A P D H$ [CTL, $1.0 \pm 0.045$; CVS, $0.52 \pm 0.038$; $\mathrm{P}<0.01$; $t=7.531$ (59); $\mathrm{F}=2.017$ ] was noticed in the hippocampus of rats submitted to CVS in comparison to the CTL. There were no changes in Ldha mRNA level as compared to CTL [CTL, 1.0 $\pm 0.048 ; \mathrm{CVS}, 0.9 \pm 0.027 ; \mathrm{P}=0.870 t=1.739$ (61); $\mathrm{F}=4.083$ ]. The results of qPCR experiments are shown in Fig. 3.

The level of amino acids. The automatic amino acid analyzer system was calibrated with an intact standard of amino acids. Amino acids in standard were at $2.5 \mu \mathrm{mol} / \mathrm{ml}$ in $0.1 \mathrm{~N} \mathrm{HCl}$, except L-cystine at $1.25 \mu \mathrm{mol} / \mathrm{ml}$. The concentrations of amino acids were determined on the basis of a three-point curve. The analysis showed a statistically significant decrease in the concentration of assayed amino acids in the prefrontal cortex of rats subjected to chronic stress. Detailed data are presented in Table III.

\section{Discussion}

After subjecting the animals to 40 days of CVS, we acknowledged a depressive-like state. Our findings

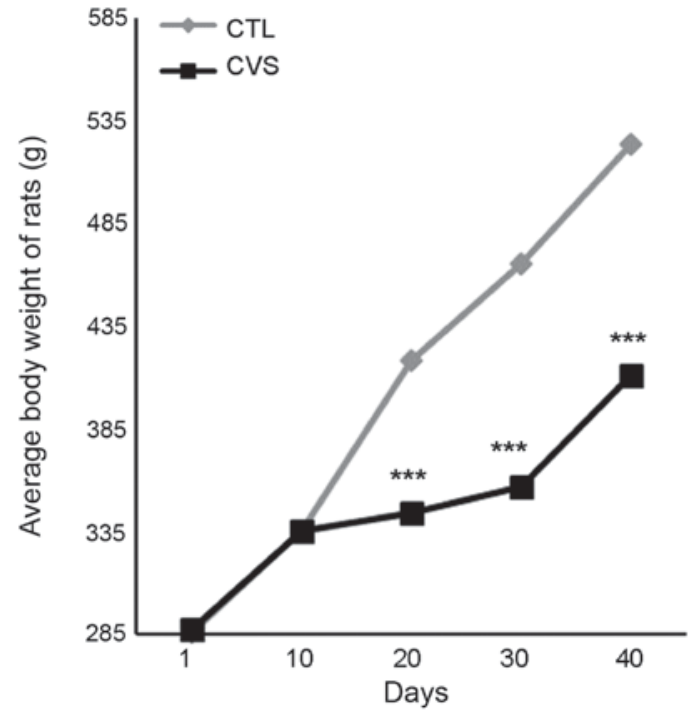

Figure 1. The impact of CVS on the body weights of rats. ${ }^{* * *} \mathrm{P}<0.001$. CTL, control; CVS, chronic variable stress.

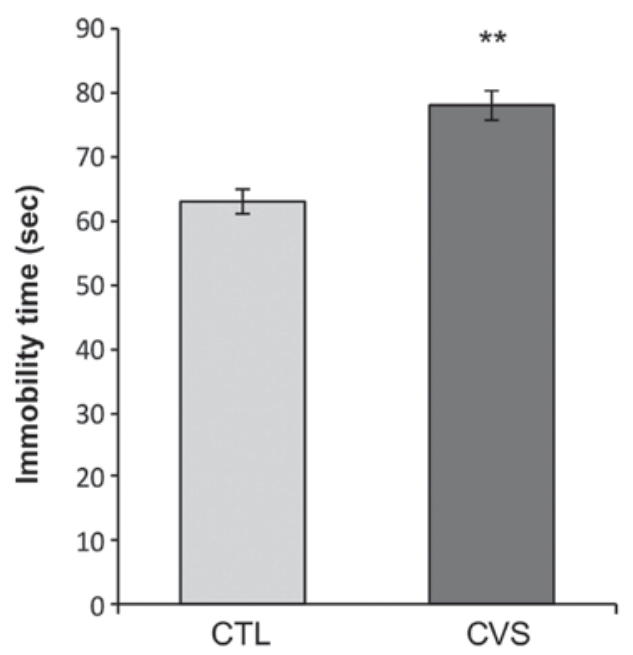

Figure 2. The impact of CVS on swimming immobility in FST. Data is displayed as mean \pm SEM. ${ }^{* *} \mathrm{P}<0.01$ by Student's t-test. FST, forced swim test; CTL, control; CVS, chronic variable stress.

demonstrated that the weight gain of the rats was significantly slower during CVS as compared to non-stressed rats. Literature data support the fact that stressed rats have a severe loss of body weight as well as behavioral alterations $(23,27)$. Emotional changes, such as exposure to stress situations can influence feeding behavior, and several studies have demonstrated that chronic exposure to stressors may alter the body weight of rats $(4,28)$. Likewise, the observed increase in the immobility time in stressed animals in the forced swimming test, currently the most popular animal model in antidepressant drug screening, which is also used in modelling of depression, suggests that these animals presented depressive behavior.

In the present study, we evaluated the changes in expression of selected genes involved in glycolytic pathway and the levels of glucogenic and neuroactive amino acids in the brain of rats exposed to CVS. We used hippocampus for molecular tests and prefrontal cortex to determine the levels of glucogenic and 
Table II. Description of the primers used in this study for reverse transcription-quantitative polymerase chain reaction.

\begin{tabular}{|c|c|c|}
\hline Gene & Primers $\left(5^{\prime}-3^{\prime}\right)$ & Accession number \\
\hline Slc $2 a 3$ & & NM_017102.2 \\
\hline Forward primer & ATGGGGACAGCGAAGGTGA & \\
\hline Reverse primer & CCCAAGGATGGCAATCAGGT & \\
\hline GAPDH & & NM_017008.4 \\
\hline Forward primer & AGTGCCAGCCTCGTCTCATA & \\
\hline Reverse primer & GATGGTGATGGGTTTCCCGT & \\
\hline Ldha & & NM_017025.1 \\
\hline Forward primer & ATCTGGATTCGGCTCGGTTC & \\
\hline Reverse primer & AACACAACTGGACCAACTGGA & \\
\hline$L d h b$ & & NM_012595.2 \\
\hline Forward primer & TTGTCTGGACAAGATGGCAAC & \\
\hline Reverse primer & TGCCGTACATTCCCTTCACC & \\
\hline Tfam & & NM_031326.1 \\
\hline Forward primer & ATCTCATCCGTCGCAGTGTG & \\
\hline Reverse primer & CTTCACAAACCCGCACGAAA & \\
\hline
\end{tabular}

neuroactive amino acids because depressed patients present alterations in these cerebral structures and relevant research most often relates to these regions of the brain. Evidence has emerged in the past decade that depression is associated with small hippocampal volumes and that this structure of the brain is particularly associated with stress-related depression (29). Thus, for the molecular tests have been used the hippocampus-a brain structure particularly associated with depression. A disturbed metabolism in the prefrontal cortex, especially dorsolateral and dorsoventral brain regions, is a frequently replicated finding in depression (30). Thus, this brain structure has been used to determine the level of amino acids.

Given its high metabolic demands and negligible intrinsic energy stores, the brain depends upon a continuous influx of substrates from the blood. Glucose is the main fuel for most cells, and its transport is tightly regulated. The current study revealed that in the hippocampus of rats subjected to CVS, the mRNA level of Slc $2 a 3$ was significantly increased as compared to CTL. These results are consistent with the hypothesis that overexpression of GLUT3 results from an increased demand of the cell for glucose (31). Detka et al (32) showed that chronic stress enhances the concentration of glucose transporters in the brain, especially in the hippocampus. These transporters are always saturated with glucose, even in states of mild hypoglycaemia, which ensures delivery of the right amount of substrate to neurons. The pathological changes such as hypoxia, chronic hypoglycemia and starvation have been shown to induce GLUT3 protein in the immature rat brain and an increase of GLUT3 mRNA in the mouse brain has also been demonstrated (33). Thus, the expression of glucose transporters can be raised in the brain as a response to an enhanced demand for glucose resulting in the support of energy metabolism. The research has shown that stress events have a significant impact on ROS generation in brain and it can cause an increase in energy demand $(2,34)$. Repeated and unpredictable stress situations increase ROS generation, which can damage a variety of cell macromolecules, including those that constitute the electron transport system, therefore disrupting mitochondrial function $(2,34,35)$. It is possible that in chronic stress, we can observe a disturbance in energy production due to mitochondrial damage by excess ROS, which may activate signaling pathways involved in cellular adaptation to various types of stress. One of these pathways is the stimulation of glucose uptake (36). The increase in GLUT3 expression associated with increased cerebral glucose utilization provides further confirmation of the central role of GLUT3. There are also assumptions that glucose uptake plays a role in regulating the balance between ROS production and scavenging, suggesting that glucose uptake must be tightly controlled to maintain cellular energy homeostasis and redox status (36).

Studies have indicated that mRNA levels of GAPDH are highly regulated in a variety of cell types in certain metabolic conditions (37-39). In our study, a significant decrease in mRNA levels of GAPDH was observed in the hippocampus of rats submitted to chronic stress in comparison to the CTL group. However, considering the fact that during stress there is an increased need for glucose, an increase in glycolysis would be expected. NAD ${ }^{+}$is required to enable the sixth step 
A

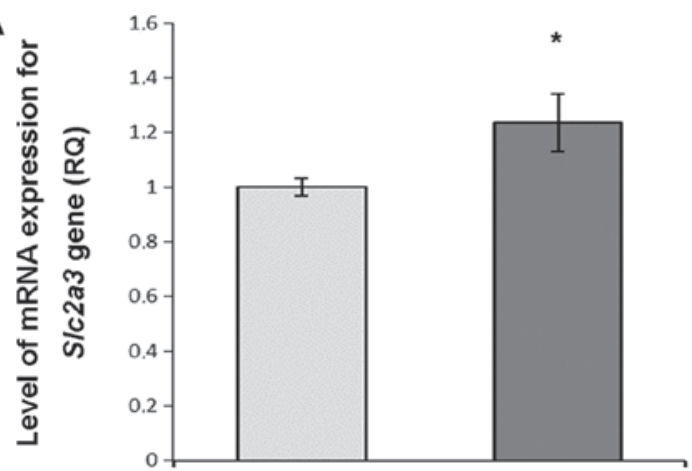

C

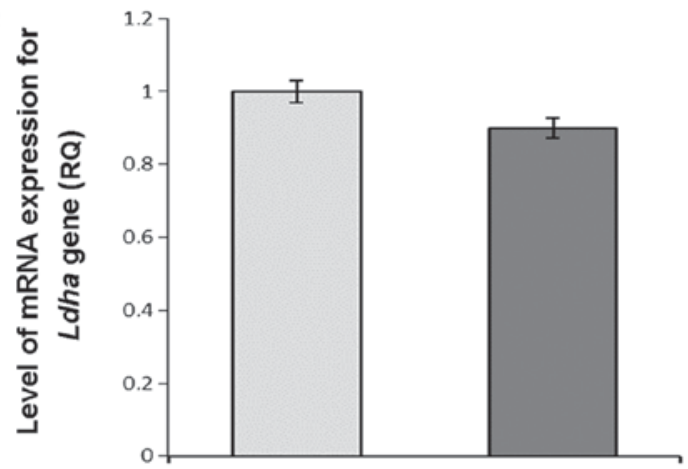

E

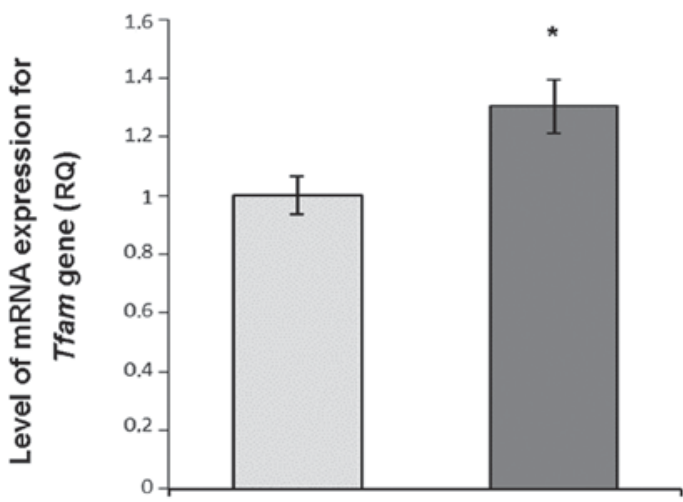

B

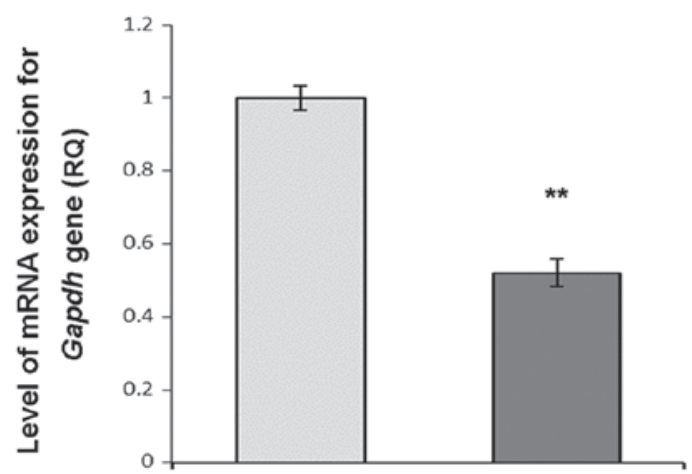

D

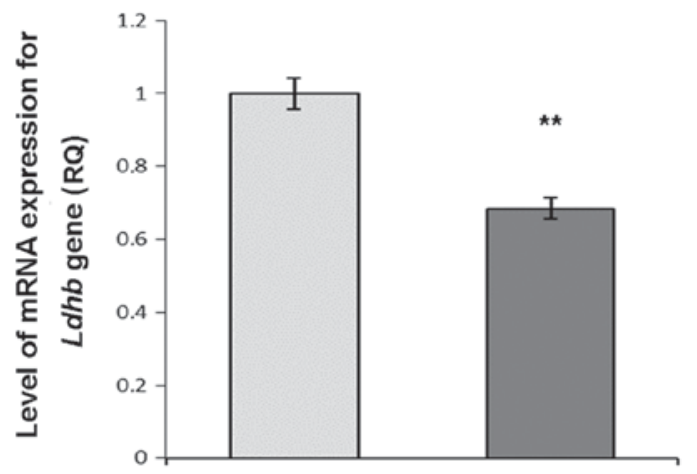

Figure 3. The impact of CVS on the levels of mRNA expression for: (A) Slc2a3 gene, (B) GAPDH gene, (C) Ldha gene, (D) Ldhb gene, (E) Tfam gene. The mRNA's expression was assayed in the hippocampus of rats using qRT-qPCR method and was assessed in regard to the control group, where the expression level (RQ) is estimated at 1 . Data is displayed as mean \pm SEM. Significance: ${ }^{*} \mathrm{P}<0.05 ;{ }^{* * *} \mathrm{P}<0.01$ by Student's $\mathrm{t}$-test. RT-qPCR, reverse transcription-quantitative polymerase chain reaction; CVS, chronic variable stress; CTL, rodents in the control group.

of glycolysis and it is usually regenerated through oxidative phosphorylation by the electron transport chain. When the oxygen supply is restricted or mitochondrial function is reduced by ROS, NAD ${ }^{+}$is regenerated from NADH by LDHA in order to maintain glycolysis, generating lactate as a by-product. It should also be emphasized that when cells are exposed to ROS, they need excessive amounts of the antioxidant cofactor NADPH, which is reduced from $\mathrm{NADP}^{+}$. In this way, the ROS excess can cause inactivation of GAPDH. This inactivation temporally routes the metabolic flux from glycolysis to the pentose phosphate pathway, allowing the cell to generate more NADPH (40). Therefore, it can be inferred that, under oxidative conditions, GAPDH can act as a switch to redirect glucose metabolism to more appropriate defensive pathways. Unfortunately, permanent inhibition of glycolysis and antioxidant defense mechanisms are ultimately overcome by the ever-increasing amount of ROS generated in the brain, which may be eliminating the possibility of cell survival. That would indicate GAPDH apoptotic function, confirmed by considerable evidence $(14,37,38,41)$. GAPDH accumulates in mitochondria during apoptosis and induces proapoptotic, increased permeability of mitochondrial membranes (42). Recent research suggests that GAPDH possesses highly diverse, non-glycolytic functions, such as nuclear, perinuclear, cytosolic, and membrane-related (14). It affects the release of $\mathrm{Ca}^{2+}$ from the mitochondria to the cytosol, thereby inducing the release of neurotransmitters $(14,43)$. The decreased mRNA level of $G A P D H$ in our study may indicate $\mathrm{Ca}^{2+}$ retention in mitochondria and consequently reduced neurotransmitter release that leads to apoptosis. GAPDH can also undergo many different oxidative modifications, which is of great interest when neurodegenerative disorders are considered. Several parallel 
Table III. The concentration of $\mathrm{G}$ and $\mathrm{N}$ amino acids in the prefrontal cortex of rats submitted to chronic variable stress. Data is displayed as mean \pm SEM.

\begin{tabular}{|c|c|c|c|c|}
\hline \multirow[b]{2}{*}{ Amino acid } & \multicolumn{2}{|c|}{ Concentration $(\mu \mathrm{mol} / \mathrm{ml}) \bar{x} \pm \mathrm{SEM}$} & \multirow[b]{2}{*}{$t$} & \multirow[b]{2}{*}{$\mathrm{F}$} \\
\hline & CTL & CVS & & \\
\hline $\begin{array}{l}\text { Alanine (ALA) } \\
\text { G N }\end{array}$ & $0.58 \pm 0.07$ & $0.21 \pm 0.01^{\mathrm{a}}$ & $5.64(25)$ & 18.19 \\
\hline $\begin{array}{l}\text { Arginine (ARG) } \\
\text { G N }\end{array}$ & $0.08 \pm 0.01$ & $0.03 \pm 0.002^{\mathrm{a}}$ & $5.19(25)$ & 17.80 \\
\hline $\begin{array}{l}\text { Aspargine (ASN) } \\
\text { G }\end{array}$ & $1.69 \pm 0.21$ & $0.53 \pm 0.03^{\mathrm{a}}$ & $5.86(25)$ & 24.71 \\
\hline $\begin{array}{l}\text { Cysteine (CYS) } \\
\text { G }\end{array}$ & $0.02 \pm 0.002$ & $0.01 \pm 0.0007^{\mathrm{a}}$ & $3.88(25)$ & 9.82 \\
\hline $\begin{array}{l}\gamma \text {-amino-butylic acid (GABA) } \\
\mathrm{N}\end{array}$ & $2.42 \pm 0.27$ & $1.31 \pm 0.03^{\mathrm{a}}$ & $4.50(25)$ & 44.39 \\
\hline $\begin{array}{l}\text { Glutamate (GLU) } \\
\text { G N }\end{array}$ & $9.98 \pm 0.98$ & $6.07 \pm 0.31^{\mathrm{a}}$ & $4.55(30)$ & 5.75 \\
\hline $\begin{array}{l}\text { Glutamine (GLN) } \\
\text { G N }\end{array}$ & $4.31 \pm 0.54$ & $2.04 \pm 0.13^{\mathrm{a}}$ & $4.50(25)$ & 13.27 \\
\hline $\begin{array}{l}\text { Glycine (GLY) } \\
\text { G N }\end{array}$ & $0.75 \pm 0.09$ & $0.2 \pm 0.01^{\mathrm{a}}$ & $5.95(25)$ & 23.03 \\
\hline $\begin{array}{l}\text { Histidine (HIS) } \\
\text { G }\end{array}$ & $0.06 \pm 0.006$ & $0.02 \pm 0.001^{\mathrm{a}}$ & $7.49(25)$ & 29.63 \\
\hline $\begin{array}{l}\text { Methionine (MET) } \\
\text { G }\end{array}$ & $0.04 \pm 0.005$ & $0.01 \pm 0.001^{\mathrm{a}}$ & $5.75(25)$ & 13.67 \\
\hline $\begin{array}{l}\text { Proline (PRO) } \\
\text { G }\end{array}$ & $0.13 \pm 0.009$ & $0.11 \pm 0.002^{\mathrm{a}}$ & $2.68(24)$ & 9.87 \\
\hline $\begin{array}{l}\text { Serine (SER) } \\
\text { G N }\end{array}$ & $0.77 \pm 0.09$ & $0.26 \pm 0.02^{\mathrm{a}}$ & $5.48(25)$ & 9.87 \\
\hline $\begin{array}{l}\text { Taurine (TAU) } \\
\mathrm{N}\end{array}$ & $5.86 \pm 0.63$ & $2.64 \pm 0.11^{\mathrm{a}}$ & $5.59(25)$ & 25.63 \\
\hline $\begin{array}{l}\text { Tyrosine (TYR) } \\
\mathrm{N}\end{array}$ & $0.07 \pm 0.003$ & $0.02 \pm 0.001^{\mathrm{a}}$ & $12.89(25)$ & 3.08 \\
\hline $\begin{array}{l}\text { Valine (VAL) } \\
\text { G }\end{array}$ & $0.07 \pm 0.007$ & $0.02 \pm 0.001^{\mathrm{a}}$ & $6.54(25)$ & 12.01 \\
\hline
\end{tabular}

${ }^{\mathrm{a} P}<0.001$ by Student's t-test. SEM, standard error of the mean; CTL, control; CVS, chronic variable stress; G, glucogenic; N, neuroactive; $t$, $\mathrm{t}$-value value of $\mathrm{t}$-test statistic; $\mathrm{F}$, degrees of freedom.

investigations indicated a relationship between GAPDH and Alzheimer's and Parkinson's diseases (42-45). It has been proven that decreased GAPDH glycolytic activity, in addition to oxidative and post-translational modifications, are distinct markers of cellular stress in pathology of these diseases that significantly impact intracellular homeostasis $(14,37,38,41)$. Taking into account GAPDH functions and locations within the cell and the above results, it can be inferred that similar mechanisms may play a role in depression.

The ability of the brain to produce and use lactate can be regulated by changes in the LDHA and LDHB gene activity, which allows the brain to optimize the use of energy and metabolism (46). The current study revealed that in the hippocampus of rats subjected to CVS, the mRNA levels of Ldha (responsible for conversion pyruvate to lactate) remained unchanged but $L d h b$ (revers reaction) levels were significantly decreased. Lactate is a substrate for the mitochondrial TCA cycle via pyruvate, and its oxidation can produce a significant amount of ATP (47). Under stress conditions, glucose biotransformation via G6PDH is defective and if the brain is under prolonged stress, neurons are more likely to depend on lactate which constitutes an alternative source of energy. The physiological significance of a decreased mRNA level of $L d h b$ in chronic stress could be an optimization use of lactate for energy production instead converting into pyruvate. These results provide data for the relative contribution of lactate probably 
driving its usage as energy fuel in conditions of chronic stress. The above results may also be related to the $\mathrm{NAD}^{+} / \mathrm{NADH}$ with excess of ROS. Because LDHB uses NAD+, also needed for NADH regeneration, the effect observed in our study may be due to the defense mechanism, enabling overproduction of NADH in response to oxidative stress. There is a hypothesis that LDH plays a role in the control of the intracellular redox status (46). According to this hypothesis, an increase of mitochondrial ROS causes mitochondrial dysfunction in the brain and leads to a metabolic shift from aerobic respiration to glycolytic metabolism, resulting in robustly increased brain lactate levels and in expression changes of the $\mathrm{LDH}$ genes. On the other hand, it has been shown that serotonin systems are involved in the control of brain glucose delivery, transport, and uptake (48). Glucose metabolism in the brain can also be disturbed by downregulation of these systems, which may result from a decrease in the mRNA level of GAPDH and occur as a consequence of the weakening of glycolysis.

TFAM is a key regulator of the maintenance and distribution of mtDNA. It is highly susceptible to the damage generated by ROS, because of its close proximity to ROS generation through the respiratory chain and its paucity of protective histones. Furthermore, there is little capacity for DNA repair in the mitochondria (49). Our study revealed a significant increase in the mRNA level of Tfam in the hippocampus of the rats subjected to CVS as compared to control. Studies show that TFAM binds preferentially to oxidatively damaged DNA containing 8-oxoguanine (8-oxo-Gua), which is one of the most abundant DNA alterations induced by exposure to ROS (50). In our previous studies, we have shown a significant increase in AP-site accumulation in isolated DNA from the hippocampus of the rats subjected to CVS, which indicates an increase in oxidative damage of DNA (2). At the same time, we noticed upregulation of $\mathrm{Oggl}$ (the gene encodes the enzyme responsible for the excision of 8-oxoguanine) as increased efficiency of DNA repair. Therefore, it can be assumed that our study is consistent with earlier results because it may indicate the contribution of mtTFA in oxidative damage through its binding to OGG1. Through the regulation of mtTFA, the maintenance of mtDNA copy number and expression are considered to be essential for preservation of mitochondrial function $(51,52)$. It was hypothesized that mtDNA and TFAM stabilize each other (53). Because ROS excess decreases the amount of mtDNA, the increased TFAM may enhance the steady state levels of mtDNA in response to oxidative stress. Considering the above mentioned results, it can be assumed that the overexpression of TFAM can prevent the decline in mtDNA, which is important for maintaining cellular functions under chronic stress. In addition, Tfam overexpression may explain the need for increased mitochondrial activity. The results we obtained in this experiment-upregulation of Slc2a3, but downregulation of GAPDH and $L d h b$, indicate a decrease in glycolysis with an increased glucose requirement. Therefore, a disruption in glucose metabolism may be a direct determinant of synaptic dysfunction, while mitochondrial changes and consequential cell death appear to be related with atrophy of the hippocampus and prefrontal cortex observed in depressed patients $(4,48,54,55)$.

In our study, it was observed a statistically significant reduction in the level of all examined amino acids in the brains of rats exposed to chronic stress. During stress exposure, the mitochondrial ATP production is disturbed. Under these circumstances, the glycolysis becomes the primary source of energy, increasing the generation of lactate. However, neither mitochondrial oxidative phosphorylation nor anaerobic glycolysis alone can produce ATP at a sufficient rate to maintain brain function. As described above, glucose is diverted towards the pentose phosphate pathway, resulting in a high antioxidant status at the expense of energy production. At contingency of high energy demand, the glucogenic amino acids can be a source of energy production in gluconeogenesis (19). Astrocytes are competent in gluconeogenesis, serving as a potential energy pathway for neurons, activated at times of stress (56). The decreased levels of amino acids may confirm the mechanism by which in brain the gluconeogenesis occurs, in order to meet the energy needs of this organ. At the same time, the above changes may provide a capacity to stimulate this pathway under chronic stress conditions.

It is worth noting that one of the stressors used in the procedure of chronic stress in rodents was the deprivation of water and food. The shortage of food and water can affect the metabolic pathways in the brain. In mammals, plasma concentration of metabolic products related to energy balance varies with a well-established dynamic, depending on whether the organism is well fed or starved (57). The brain engages in gluconeogenesis to convert glucogenic amino acids into glucose for metabolism. The body's glycogen stores are consumed in about $24 \mathrm{~h}$ (58). Unless we're talking about the case of long-term starvation, brain energy requirements are covered in part by the metabolism of ketone bodies (58). Studies showed that rodents are physiologically equipped to tolerate acute food and water deprivation for periods of as long as $24 \mathrm{~h}$ without overt signs of physiologic distress or behavioral abnormalities (59). Therefore, we assume that short-term deprivation of water and food $(24 \mathrm{~h})$ did not significantly affect the energy metabolism of the rats brain due to adaptive properties. We can therefore consider the deprivation of water and food as appropriate stress factors, especially that patients suffering from stress-related depression show concurrent disordered eating behaviors; they consume excessive amounts of food or they use starvation as a means of coping with the seemingly intolerable feelings of depression. However, it is not clear whether this data can be directly related to people; it is unknown whether short-term starvation of a few days' duration leads to reduced brain glucose metabolism in human. Rodents have endogenous nycthemeral rhythms that make them particularly adaptable to once-daily occurrences, such as food or water access. During water deprivation in rats, the increases in plasma osmolality is only a modest approximately $2 \%$ after $24 \mathrm{~h}$; the corresponding decreases in plasma volume is approximately $4 \%$ and may be less than in humans (59). Studies showed that the human brain adapts to the changes in energy supply as 3 days following initiation of starvation, at which time ketone bodies account for approximately one-fourth of the cerebral energy requirements (58).

A number of assumption regarding the molecular mechanism of neurodegeneration involves the neuroactive amino acids (60). Aspartate and glutamate are the major excitatory neurotransmitters in the brain, whereas, glycine, taurine and $\gamma$-aminobutyric acid are inhibitory. Taurine has 
also a protective role in the brain. In addition, glutamine is a precursor molecule for glutathione, which protects against ROS toxicity (19). Amino acids such as tryptophan, tyrosine, histidine, and arginine are used by the brain for the synthesis of various neurotransmitters and neuromodulators (22). Abnormal metabolism of neuroactive amino acids has been demonstrated in the plasma of patients with major depression and also in the brain samples of rats in depression model (61-63). Ni et al (61) showed that tyrosine, glutamine and proline were significantly downregulated, while glycine was upregulated in the cerebella of depressed rats. The decrease of neuroactive amino acids levels observed in our study can result from a close relationship between the brain activity, neurotransmission, energy requirements, and glucose utilization. During activation, glutamate uptake into astrocytes leads to the increased glucose use and lactate production, therefore, it can be subsequently used by neurons to meet their energy needs (56). Glutamate and aspartate, the major excitatory neurotransmitters in the brain, do not cross the BBB and are synthesized from glucose. Therefore, the observed reduction in their level provides further support for the hypothesis of redirecting glucose metabolism to defense pathways in conditions of chronic stress. The changes in concentration of neuroactive amino acids could be also a consequence of an imbalance between excitation and inhibition, as well as alterations of ion channels during stress. NMDA receptors are highly permeable to calcium ions which enter cells via NMDA receptor channels, leading to mitochondrial damage. Recent studies provide the impaired or reduced glutamatergic neurotransmission in neurological diseases involving hypofunction of NMDA (64). The above data are consistent with the reduction of GAPDH mRNA levels noted in our study, which can provide $\mathrm{Ca}^{2+}$ retention in mitochondria and consequently reduced neurotransmitter release.

In conclusion, the changes in gene expression and amino acids levels confirm that the brain energy metabolism involves complex cellular and molecular mechanisms, which are necessary to meet the increased energy requirements of the brain during stress-related depression. The alterations indicate the support of the energy metabolism by stimulation of gluconeogenesis pathway and redirection of glucose metabolism to appropriate defensive pathways under chronic stress conditions. The overexpression of Tfam may confirm the oxidative damage of DNA and, at the same time, it may indicate an increased ability to maintain mitochondrial function as potential adaptive response. We assume that these changes are coupled with various signaling mechanisms, which need to be controlled by the cell to avoid oxidative stress. Our findings provide a novel insight into the biochemical and molecular events that lead to the development of depression under chronic stress conditions and they may identify novel targets for therapeutic intervention.

\section{Acknowledgments}

Not applicable.

\section{Funding}

The present study was supported by Funds for Statutory Activity of Medical University of Lublin, Poland (grant no. DS 38/2015-2016). This research did not receive any specific grant from funding agencies in the public, commercial, or not-for-profit sectors.

\section{Availability of data and materials}

The datasets used and/or analyzed during the current study are available from the corresponding author on reasonable request.

\section{Authors' contributions}

MH designed and directed the experiment, wrote the manuscript, analyzed the data, performed the experiments, corresponding author; DN-C performed the analysis of amino acids, edited the manuscript; AK and MO performed the molecular study; MI analyzed and collected the data; MG-G and IP-C performed the statistical analysis; KP performed the experiments; BŚ supervised the molecular study, EP supervised the in vivo experiments, JD supervised the analysis of amino acids; BŚ, EP and JD critically revised the final version of the manuscript. All authors read and approved the final manuscript.

\section{Ethics approval and consent to participate}

All procedures were conducted in accordance with the European Communities Council Directive of 22 September 2010 (2010/63/EU) and Polish legislation acts concerning animal experimentations. The experimental procedures and protocols were approved by the First Local Ethics Committee at the Medical University of Lublin.

\section{Patient consent for publication}

Not applicable.

\section{Competing interests}

The authors declare that they have no competing interests.

\section{References}

1. Bansal Y and Kuhad A: Mitochondrial dysfunction in depression. Curr Neuropharmacol 14: 610-618, 2016.

2. Herbet M, Korga A, Gawrońska-Grzywacz M, Izdebska M, Piatkowska-Chmiel I, Poleszak E, Wróbel A, Matysiak W, Jodłowska-Jędrych B and Dudka J: Chronic variable stress is responsible for lipid and DNA oxidative disorders and activation of oxidative stress response genes in the brain of rats. Oxid Med Cell Longev 2017: 7313090, 2017.

3. Khan S and Khan RA: Chronic stress leads to anxiety and depression. Ann Psychiatry Ment Health 5: 1091, 2017.

4. Tagliari B, Noschang CG, Ferreira AG, Ferrari OA, Feksa LR, Wannmacher CM, Dalmaz C and Wyse AT: Chronic variable stress impairs energy metabolism in prefrontal cortex and hippocampus of rats: Prevention by chronic antioxidant treatment. Metab Brain Dis 25: 169-176, 2010.

5. Mergenthaler P, Lindauer U, Dienel GA and Meisel A: Sugar for the brain: The role of glucose in physiological and pathological brain function. Trends Neurosci 36: 587-597, 2013.

6. Detka J, Kurek A, Kucharczyk M, Głombik K, Basta-Kaim A Kubera M,Lasoń W and Budziszewska B: Brain glucose metabolism in an animal model of depression. Neuroscience 295: 198-208, 2015.

7. Ishida A, Noda Y and Ueda T: Synaptic vesicle-bound pyruvate kinase can support vesicular glutamate uptake. Neurochem Res 34: 807-818, 2009. 
8. Rezin GT, Cardoso MR, Gonçalves CL, Scaini G, Fraga DB, Riegel RE, Comim CM, Quevedo J and Streck EL: Inhibition of mitochondrial respiratory chain in brain of rats subjected to an experimental model of depression. Neurochem Int 53: 395-400, 2008.

9. Bélanger M, Allaman I and Magistretti PJ: Brain energy metabolism: Focus on astrocyte-neuron metabolic cooperation. Cell Metab 14: 724-738, 2011.

10. Griffin JW and Bradshaw PC: Amino acid catabolism in Alzheimer's disease brain: Friend or foe? Oxid Med Cell Longev 2017: 5472792, 2017.

11. Chiefari E, Foti DP, Sgarra R, Pegoraro S, Arcidiacono B, Brunetti FS, Greco M and Manfioletti G: Transcriptional regulation of glucose metabolism: The emerging role of the HMGA1 chromatin factor. Front Endocrinol (Lausanne) 9 357, 2018.

12. Shah K, Desilva S and Abbruscato T: The role of glucose transporters in brain disease: Diabetes and alzheimer's disease. Int J Mol Sci 13: 12629-12655, 2012.

13. Camandola S and Mattson MP: Brain metabolism in health, aging, and neurodegeneration. EMBO J 36: 1474-1492, 2017.

14. Butterfield DA, Hardas SS and Lange ML: Oxidatively modified glyceraldehyde-3-phosphate dehydrogenase (GAPDH) and Alzheimer's disease: Many pathways to neurodegeneration. J Alzheimers Dis 20: 369-393, 2010.

15. Kasashima $\mathrm{K}$ and Endo $\mathrm{H}$ : Interaction of human mitochondrial transcription factor A in mitochondria: Its involvement in the dynamics of mitochondrial DNA nucleoids. Genes Cells 20 1017-1027, 2015

16. Campbell CT, Kolesar JE and Kaufman BA: Mitochondrial transcription factor A regulates mitochondrial transcription initiation, DNA packaging, and genome copy number. Biochim Biophys Acta 1819: 921-929, 2012.

17. Murakami T, Yamane H, Tomonaga T and Furuse M: Forced swimming and imipramine modify plasma and brain amino acid concentrations in mice. Eur J Pharmacol 602: 73-77, 2009

18. Nagasawa M, Ogino Y, Kurata K, Otsuka T, Yoshida J, Tomonaga $\mathrm{S}$ and Furuse M: Hypothesis with abnormal amino acid metabolism in depression and stress vulnerability in Wistar Kyoto rats. Amino Acids 43: 2101-2111, 2012.

19. Yip J, Geng X, Shen J and Ding Y: Cerebral gluconeogenesis and diseases. Front Pharmacol 7: 521, 2017.

20. Heimburger DC and Ard JD: Handbook of Clinical Nutrition. 4th edition, Elsevier Inc., 2006, https://doi. org/10.1016/C2009-0-45871-9.

21. Ghosh A, Cheung YY, Mansfield BC and Chou JY: Brain contains a functional glucose-6-phosphatase complex capable of endogenous glucose production. J Biol Chem 280: 11114-11119, 2005 .

22. Maher T: The role of amino acid precursors on neurotransmission. Eur J Pharmacol 668: e8, 2011.

23. Gamaro GD, Manoli LP, Torres IL, Silveira R and Dalmaz C: Effects of chronic variate stress on feeding behavior and on monoamine levels in different rat brain structures. Neurochem Int 42: 107-114, 2003.

24. Zhao Z, Wang W, Guo H and Zhou D: Antidepressant-like effect of liquiritin from Glycyrrhiza uralensis in chronic variable stress induced depression model rats. Behav Brain Res 194: 108-113, 2008

25. Porsolt RD, Bertin A and Jalfre M: Behavioral despair in mice: A primary screening test for antidepressants. Arch Int Pharmacodyn Ther 229: 327-336, 1977

26. Iłżecka J, Stelmasiak Z, Solski J, Wawrzycki S and Szpetnar M: Plasma amino acids concentration in amyotrophic lateral sclerosis patients. Amino Acids 25: 69-73, 2003.

27. Bekris S, Antoniou K, Daskas S and Papadopoulou-Daifoti Z Behavioural and neurochemical effects induced by chronic mild stress applied to two different rat strains. Behav Brain Res 161: 45-59, 2005

28. Packard AE, Ghosal S, Herman JP, Woods SC and Ulrich-Lai YM: Chronic variable stress improves glucose tolerance in rats with sucrose-induced prediabetes. Psychoneuroendocrinology 47: 178-188, 2014.

29. MacQueen G and Frodl T: The hippocampus in major depression: Evidence for the convergence of the bench and bedside in psychiatric research? Mol Psychiatry 16: 252-264, 2011.

30. Pandya M, Altinay M, Malone DA Jr and Anand A: Where in the brain is depression? Curr Psychiatry Rep 14: 634-642, 2012.

31. Dienel GA: Fueling and imaging brain activation. ASN Neuro 4: pii: e00093, 2012.
32. Detka J, Kurek A, Basta-Kaim A, Kubera M, Lasoń W and Budziszewska B: Elevated brain glucose and glycogen concentrations in an animal model of depression. Neuroendocrinology 100 : 178-190, 2014

33. Khayat ZA, McCal AL and Klip A: Unique mechanism of GLUT3 glucose transporter regulation by prolonged energy demand: increased protein half-life. Biochem J 333: 713-718, 1998.

34. Fontella FU, Siqueira IR, Vasconcellos AP, Tabajara AS, Netto CA and Dalmaz C: Repeated restraint stress induces oxidative damage in rat hippocampus. Neurochem Res 30: 105-111, 2005

35. Lucca G, Comim CM, Valvassori SS, Réus GZ, Vuolo F, Petronilho F, Gavioli EC, Dal-Pizzol F and Quevedo J: Increased oxidative stress in submitochondrial particles into the brain of rats submitted to the chronic mild stress paradigm. J Psychiatr Res 43: 864-869, 2009.

36. Liemburg-Apers DC, Willems PH, Koopman WJ and Grefte S: Interactions between mitochondrial reactive oxygen species and cellular glucose metabolism. Arch Toxicol 89: 1209-1226, 2015.

37. Ishitani R, Kimura M, Sunaga K, Katsube N, Tanaka M and Chuang DM: An antisense oligodeoxynucleotide to glyceraldehyde-3-phosphate dehydrogenase blocks age-induced apoptosis of mature cerebrocortical neurons in culture. J Pharmacol Exp Ther 278: 447-454, 1996.

38. Ishitani R, Sunaga K, Hirano A, Saunders P, Katsube N and Chuang DM: Evidence that glyceraldehyde-3-phosphate dehydrogenase is involved in age-induced apoptosis in mature cerebellar neurons in culture. J Neurochem 66: 928-935, 1996.

39. Vilà MR, Nicolás A, Morote J, de Torres I and Meseguer A: Increased glyceraldehyde-3-phosphate dehydrogenase expression in renal cell carcinoma identified by RNA-based, arbitrarily primed polymerase chain reaction. Cancer 89: 152-164, 2000

40. Grant CM: Metabolic reconfiguration is a regulated response to oxidative stress. J Biol 7: 1, 2008.

41. Chuang DM, Hough C and Senatorov VV: Glyceraldehyde-3-phosphate dehydrogenase, apoptosis, and neurodegenerative diseases. Annu Rev Pharmacol Toxicol 45: 269-290, 2005

42. Tarze A, Deniaud A, Le Bras M, Maillier E, Molle D, Larochette N, Zamzami N, Jan G, Kroemer G and Brenner C: GAPDH, a novel regulator of the pro-apoptotic mitochondrial membrane permeabilization. Oncogene 26: 2606-2620, 2007.

43. Mazzola JL and Sirover MA: Alteration of intracellular structure and function of glyceraldehyde-3-phosphate dehydrogenase: A common phenotype of neurodegenerative disorders? Neurotoxicology 23: 603-609, 2002.

44. Sirover MA: Role of the glycolytic protein, glyceraldehyde-3-phosphate dehydrogenase, in normal cell function and in cell pathology. J Cell Biochem 66: 133-140, 1997.

45. Tatton WG, Chalmers-Redman RM, Elstner M, Leesch W, Jagodzinski FB, Stupak DP, Sugrue MM and Tatton NA: Glyceraldehyde-3-phosphate dehydrogenase in neurodegeneration and apoptosis signaling. J Neural Transm Suppl: 77-100, 2000.

46. Ross JM, Öberg J, Brené S, Coppotelli G, Terzioglu M, Pernold K, Goiny M, Sitnikov R, Kehr J, Trifunovic A, et al: High brain lactate is a hallmark of aging and caused by a shift in the lactate dehydrogenase A/B ratio. Proc Natl Acad Sci USA 107: 20087-20092, 2010

47. Schurr A: Lactate: The ultimate cerebral oxidative energy substrate? J Cereb Blood Flow Metab 26: 142-152, 2006.

48. Mosconi L, Pupi A and De Leon MJ: Brain glucose hypometabolism and oxidative stress in preclinical Alzheimer's disease. Ann N Y Acad Sci 1147: 180-195, 2008.

49. Nakanishi $\mathrm{H}$ and $\mathrm{Wu} \mathrm{Z}$ : Microglia-aging: Roles of microglial lysosome- and mitochondria-derived reactive oxygen species in brain aging. Behav Brain Res 201: 1-7, 2009.

50. Yoshida Y, Izumi H, Ise T, Uramoto H, Torigoe T, Ishiguchi H, Murakami T, Tanabe M, Nakayama Y, Itoh H, et al: Human mitochondrial transcription factor A binds preferentially to oxidatively damaged DNA. Biochem Biophys Res Commun 295: 945-951, 2002

51. Jeng JY, Yeh TS, Lee JW, Lin SH, Fong TH and Hsieh RH: Maintenance of mitochondrial DNA copy number and expression are essential for preservation of mitochondrial function and cell growth. J Cell Biochem 103: 347-357, 2008.

52. Toki N, Kagami S, Kurita T, Kawagoe T, Matsuura Y, Hachisuga T, Matsuyama A, Hashimoto H, Izumi H and Kohno K: Expression of mitochondrial transcription factor A in endometrial carcinomas: Clinicopathologic correlations and prognostic significance. Virchows Arch 456: 387-393, 2010. 
53. Kang D, Kim SH and Hamasaki N: Mitochondrial transcription factor A (TFAM): Roles in maintenance of mtDNA and cellular functions. Mitochondrion 7: 39-44, 2007.

54. Lee AL, Ogle WO and Sapolsky RM: Stress and depression: Possible links to neuron death in the hippocampus. Bipolar Disord 4: 117-128, 2002.

55. Gałecki P, Florkowski A, Mrowicka M, Pietras T and Gałecka E: Calcium ions, glutaminate acid, hypothalamic-pituitary-adrenal axis, calcium dependent ATP-ase as causes of oxidative damage in depression patients (part II). Pol Merkur Lekarski 24: 72-75, 2008 (In Polish)

56. Magistretti PJ and Pellerin L: Cellular mechanisms of brain energy metabolism and their relevance to functional brain imaging. Philos Trans R Soc Lond B Biol Sci 354: 1155-1163, 1999.

57. Hasselbalch SG, Knudsen GM, Jakobsen J, Hageman LP, Holm S and Paulson OB: Brain metabolism during short-term starvation in humans. J Cereb Blood Flow Metab 14: 125-131, 1994.

58. White $\mathrm{H}$ and Venkatesh B: Clinical review: Ketones and brain injury. Crit Care 15: 219, 2011.

59. Rowland NE: Food or fluid restriction in common laboratory animals: Balancing welfare considerations with scientific inquiry. Comp Med 57: 149-160, 2007.
60. Kay GW, Verbeek MM, Furlong JM, Willemsen MA and Palmer DN: Neuropeptide changes and neuroactive amino acids in CSF from humans and sheep with neuronal ceroid lipofuscinoses (NCLs, Batten disease). Neurochem Int 55: 783-788, 2009.

61. Ni Y, Su M, Lin J, Wang X, Qiu Y, Zhao A, Chen T and Jia W: Metabolic profiling reveals disorder of amino acid metabolism in four brain regions from a rat model of chronic unpredictable mild stress. FEBS Lett 582: 2627-2636, 2008.

62. Xu HB, Zhang RF, Luo D, Zhou Y, Wang Y, Fang L, Li WJ, Mu J, Zhang L, Zhang Y and Xie P: Comparative proteomic analysis of plasma from major depressive patients: Identification of proteins associated with lipid metabolism and immunoregulation. Int J Neuropsychopharmacol 15: 1413-1425, 2012.

63. Shao WH, Chen JJ, Fan SH, Lei Y, Xu HB, Zhou J, Cheng PF, Yang YT, Rao CL, Wu B, et al: Combined metabolomics and proteomics analysis of major depression in an animal model: Perturbed energy metabolism in the chronic mild stressed rat cerebellum. OMICS 19: 383-392, 2015.

64. Kantrowitz JT and Javitt DC: N-methyl-d-aspartate (NMDA) receptor dysfunction or dysregulation: The final common pathway on the road to schizophrenia? Brain Res Bull 83: $108-121,2010$ 